\title{
First Presenting Symptom Related to Disease Unknown
}

National Cancer Institute

\section{Source}

National Cancer Institute. First Presenting Symptom Related to Disease Unknown. NCI

Thesaurus. Code C160173.

The first presenting symptom related to the disease is unknown. 